\title{
Exact and Approximate Strategies for Symmetry Reduction in Model Checking
}

\author{
Alastair F. Donaldson ${ }^{\star}$ and Alice Miller \\ Department of Computing Science \\ University of Glasgow \\ Glasgow, Scotland. \\ \{ally, alice\}@dcs.gla.ac.uk
}

\begin{abstract}
Symmetry reduction techniques can help to combat the state space explosion problem for model checking, but are restricted by the hard problem of determining equivalence of states during search. Consequently, existing symmetry reduction packages can only exploit full symmetry between system components, as checking the equivalence of states is straightforward in this special case. We present a framework for symmetry reduction with an arbitrary group of structural symmetries. By generalising existing techniques for efficiently exploiting symmetry, and introducing an approximate strategy for use with groups for which fast, exact strategies are not available, our approach allows for significant state-space reduction with minimal time overhead. We show how computational group theoretic techniques can be used to analyse the structure of a symmetry group so that an appropriate symmetry reduction strategy can be chosen, and we describe a symmetry reduction package for the SPIN model checker which interfaces with the computational algebra system GAP. Experimental results on a variety of Promela models illustrate the effectiveness of our methods.
\end{abstract}

\section{Keywords}

Promela/SPIN; model checking; symmetry; computational group theory; GAP

\section{Introduction}

Symmetry reduction techniques can help to combat the state space explosion problem when model checking systems with replicated structure. Replication of components in a concurrent system frequently induces replication, or symmetry, in a Kripke structure modelling the system, which partitions the states of the model into equivalence classes. To model check temporal logic properties it is often sufficient to search one state per equivalence class, potentially resulting in more efficient verification. Given a symmetry group $G$, a common approach to ensure that equivalent states are recognised during search is to convert each

\footnotetext{
^ Supported by the Carnegie Trust for the Universities of Scotland.
} 
newly encountered state $s$ into $\min [s]_{G}$ the smallest state in its equivalence class (under a suitable total ordering) before it is stored. However, the problem of computing $\min [s]_{G}$ for an arbitrary group, called the constructive orbit problem $(\mathrm{COP})$, is NP-hard [5].

Existing symmetry reduction packages, such as SymmSpin [1] and SMC [19], are limited as they can only exploit full symmetry between identical components of a system. Such symmetry arises in systems where all components of the same type are interchangeable, and has been of primary interest since the COP can be efficiently solved in this special case. However, many other kinds of symmetry commonly occur in models of concurrent systems with a regular structure. For example, cyclic/dihedral groups are typically associated with systems which have uni-/bi-directional ring structures and wreath product groups occur when dealing with tree topologies.

In this paper we generalise existing techniques for efficiently exploiting symmetry, and give an approximate strategy for use with symmetry groups for which fast, exact strategies cannot be found. We use computational group theory to automatically determine the structure of a group before search so that an appropriate symmetry reduction strategy can be chosen, and give encouraging experimental results to support our techniques using TopSPIN, a new symmetry reduction package for the SPIN model checker [15] which interfaces with the GAP computational algebra system [13]. In the spirit of previous works on symmetry reduction [5,10-12], we use a simple model of computation when reasoning about concurrent systems. We then illustrate the problems associated with extending this simple model to apply to Promela specifications, where full symmetry reduction may no longer be guaranteed.

\section{Symmetry in Model Checking}

\subsection{Model of Computation}

We use a simple model of computation to represent the computation of a system comprised of $n$ communicating components, interleaving concurrently [10,11]. Let $I=\{1,2, \ldots, n\}$ be the set of component identifiers, and for some $k>0$, let $\{1,2, \ldots, k\}$ denote the possible local states of the components. A Kripke structure is a pair $\mathcal{M}=(S, R)$, where $S \subseteq\{1,2, \ldots, k\}^{n}$, is a non-empty set of states, and $R \subseteq S \times S$ is a total transition relation. The lexicographical ordering of vectors in $\{1,2, \ldots, k\}^{n}$ provides a total ordering on $S$. If $s=\left(l_{1}, l_{2}, \ldots, l_{n}\right) \in$ $S$ then we use $s(i)$ to denote $l_{i}$, the local state of component $i$.

This model of computation allows us to reason about concurrent systems consisting of processes and channels, since a positive integer can be assigned to each valuation of the local variables of a process or the contents of a channel. We assume that the local variables of components do not refer to component identifiers. We discuss the implications of lifting this assumption in Section 5.1. 


\subsection{Group Theoretic Notation}

We assume some knowledge of basic group theory, but recap some notation here. Let $G$ be a group, and let $\alpha_{1}, \alpha_{2}, \ldots, \alpha_{n} \in G$. The smallest subgroup of $G$ containing the elements $\alpha_{1}, \ldots, \alpha_{n}$ is denoted $\left\langle\alpha_{1}, \alpha_{2}, \ldots, \alpha_{n}\right\rangle$, and is called the subgroup generated by $\alpha_{1}, \alpha_{2}, \ldots, \alpha_{n}$. The elements $\alpha_{i}(1 \leq i \leq n)$ are called generators for this subgroup. Let $X=\left\{\alpha_{1}, \ldots, \alpha_{n}\right\}$ be a finite subset of $G$. Then we use $\langle X\rangle$ to denote $\left\langle\alpha_{1}, \ldots, \alpha_{n}\right\rangle$, the subgroup generated by $X$. Let $H$ be a subgroup of $G$, denoted $H \leq G$, and let $\alpha \in G$. The set $H \alpha=\{\beta \alpha: \beta \in H\}$ is called a (right) coset of $H$ in $G$. The set of all cosets of $H$ in $G$ partitions $G$ into disjoint equivalence classes.

Let $I=\{1,2, \ldots, n\}$ for some $n>0$. The set of all permutations of $I$ forms a group under composition of mappings, denoted $S_{n}$ (the symmetric group on $n$ points). If $J \subseteq I$ and $\alpha \in S_{n}$, then $\alpha(J)=\{\alpha(i): i \in J\}$. For $H \leq S_{n}$ and $\alpha \in S_{n}$, we define $\operatorname{moved}(H)=\{i \in I: \beta(i) \neq i$ for some $\beta \in H\}$, and $\operatorname{moved}(\alpha)=\{i \in I: \alpha(i) \neq i\}$. For $i \in I$, the stabiliser of $i$ under $H$ is the subgroup $\operatorname{stab}_{H}(i)=\{\alpha \in H: \alpha(i)=i\}$, and the orbit of $i$ under $H$ is the set $\operatorname{orb}_{H}(i)=\{\alpha(i): \alpha \in H\}$. The orbit $\operatorname{orb}_{H}(i)$ is non-trivial if $\left|\operatorname{orb}_{H}(i)\right|>1$, and $H$ is said to act transitively on $I$ if it induces a single orbit.

\subsection{Symmetry Reduction}

Let $\mathcal{M}=(S, R)$ be a Kripke structure, and let $\alpha \in S_{n}$ (the group of all permutations of the set $\{1,2, \ldots, n\})$. Then $\alpha$ acts on a state $s=\left(l_{1}, l_{2}, \ldots, l_{n}\right) \in S$ in the following way: $\alpha(s)=\left(l_{\alpha^{-1}(1)}, l_{\alpha^{-1}(2)}, \ldots, l_{\alpha^{-1}(n)}\right)$. If $(\alpha(s), \alpha(t)) \in R \forall(s, t) \in$ $R, \alpha$ is an automorphism of $\mathcal{M}$. The set of all automorphisms of $\mathcal{M}$ forms a group $\operatorname{Aut}(\mathcal{M}) \leq S_{n}$ under composition of mappings.

A subgroup $G \leq A u t(\mathcal{M})$ induces an equivalence relation $\equiv_{G}$ on the states of $\mathcal{M}$ thus: $s \equiv_{G} t \Leftrightarrow s=\alpha(t)$ for some $\alpha \in G$. The equivalence class under $\equiv_{G}$ of a state $s \in S$, denoted $[s]$, is called the orbit of $s$ under the action of $G$ (so $G$ induces orbits on both the set $I$ of component identifiers and the set $S$ of states), and $\min [s]_{G}$ denotes the smallest element of $[s]_{G}$ under the total ordering discussed in Section 2.1. The quotient Kripke structure for $\mathcal{M}$ with respect to $G$ is a pair $\mathcal{M}_{G}=\left(S_{G}, R_{G}\right)$ where $S_{G}=\left\{\min [s]_{G}: s \in S\right\}$, and $R_{G}=\left\{\left(\min [s]_{G}, \min [t]_{G}\right):(s, t) \in R\right\}$. In general $\mathcal{M}_{G}$ is a smaller structure than $\mathcal{M}$, but $\mathcal{M}_{G}$ and $\mathcal{M}$ are equivalent in the sense that they satisfy the same set of logic properties which are invariant under the group $G$ (that is, properties which are "symmetric" with respect to $G$ ). For a proof of the following theorem, together with details of the temporal logic $C T L^{*}$, see [6].

Theorem 1. Let $\mathcal{M}$ be a Kripke structure, $G$ a subgroup of $A u t(\mathcal{M})$ and $\phi$ a $C T L^{*}$ formula. If $\phi$ is invariant under $G$ then

$$
\mathcal{M}, s \models \phi \Leftrightarrow \mathcal{M}_{G}, \min [s]_{G} \models \phi
$$

Thus by choosing a suitable symmetry group $G$, model checking can be performed over $\mathcal{M}_{G}$ instead of $\mathcal{M}$, often resulting in considerable savings in memory 
and verification time $[2,11]$. Algorithm 1, adapted from [16], explores a quotient Kripke structure given an initial state $s_{0}$. An extension of this algorithm for on-the-fly model checking of $L T L$ properties using a nested depth first search is presented in [1].

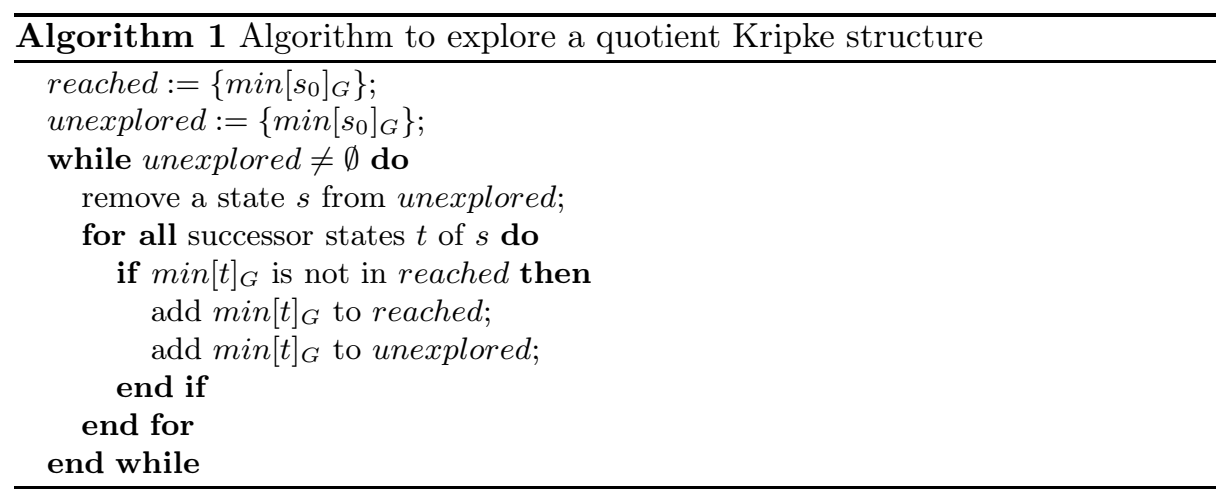

In practice, for an arbitrary group $G$, it may be infeasible to implement the function min exactly. In such cases the requirements of min can be relaxed so that $\min [s]_{G}$ yields some state $t \in[s]_{G}$ with $t \leq s$. This does not compromise the safety of symmetry reduced model checking since at least one state per orbit is searched, but does not result in memory-optimal verification. However, an efficient implementation of min which maps any element $s$ to one of a small number of orbit representatives can result in fast verification, maintaining a significant reduction in model states (this use of multiple representatives is employed in e.g. $[2,5])$. We refer to such an implementation of min as an approximate symmetry reduction strategy, whereas a true implementation of min is an exact strategy. Note that exact verification results are still obtained using an approximate symmetry reduction strategy, if enough memory is available.

Throughout the rest of the paper, let $G$ be a subgroup of $\operatorname{Aut}(\mathcal{M})$, where $\mathcal{M}=(S, R)$ is a Kripke structure which models a concurrent system comprised of $n$ components with identifiers $I=\{1,2, \ldots, n\}$.

\subsection{Symmetry Detection}

In this paper, we are concerned with techniques for exploiting component symmetries during model checking, rather than detecting symmetry before search. Structural symmetries of a model $\mathcal{M}$ are typically inferred by extracting a communication graph from the initial specification. The vertex set of this graph is the set $I$, representing the components of the system. Provided that the specification obeys certain restrictions so that components of the same type are not explicitly distinguished, automorphisms of the communication graph induce automorphisms of $\mathcal{M}$. Since the communication graph is typically small, these 
automorphisms can be computed automatically using a package such as saucy [7]. Practical examples of communication graphs include the static channel diagram of a Promela specification, which has been introduced in previous work [8], and the coloured hypergraph [5] of a shared variable concurrent program.

For illustration, throughout the paper we consider a system with a threetiered architecture consisting of a database, a layer of server components, and a layer of client components, each of which communicates with exactly one server. Figure 1 shows a possible communication graph for this system, which we assume has been extracted from a specification of the system by some symmetry detection tool. Let $\mathcal{M}_{3 T}$ be a model of the system. Using the saucy program, we compute generators for $G_{3 T}$, the automorphism group of the communication graph:

$$
\begin{aligned}
G_{3 T}= & \langle(12),(23),(45),(56),(78),(89),(1011), \\
& (1213)(14)(25)(36),(1314)(47)(58)(6 \text { 9 })\rangle .
\end{aligned}
$$

Note that the last two elements of the generating set of $G_{3 T}$ are products of transpositions. We assume that $G_{3 T} \leq A u t\left(\mathcal{M}_{3 T}\right)$, and will use this group and its subgroups as examples to illustrate some of our techniques.

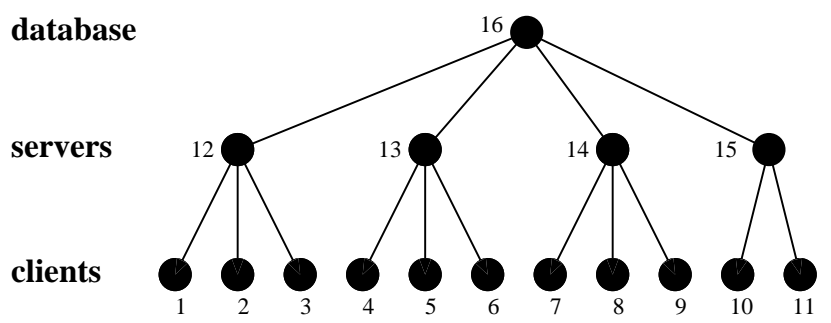

Fig. 1. Communication structure for a three-tiered architecture.

\section{Exploiting Basic Symmetry Groups}

\subsection{Enumerating Small Groups}

The most obvious strategy for computing $\min [s]_{G}$ is to consider each state in $[s]_{G}$, and return the smallest. This can be achieved by enumerating the elements $\alpha(s), \forall \alpha \in G$. If $G$ is small then this strategy is feasible in practice, and provides an exact symmetry reduction strategy. The SymmSpin package provides an enumeration strategy for fully symmetric groups, which is optimised by generating permutations incrementally by composing successive transpositions. This is more efficient than applying permutations to $s$ directly. 
We generalise this optimisation for arbitrary groups using stabiliser chains. A stabiliser chain for $G$ is a series of subgroups of the form $G=G^{(1)} \geq G^{(2)} \geq$ $\cdots \geq G^{(k)}=\{i d\}$, for some $k>1$, where $G^{(i)}=\operatorname{stab}_{G^{(i-1)}}(x)$ for some $x \in$ moved $\left(G^{(i-1)}\right)(2 \leq i \leq k)$. If $U^{(i)}$ is a set of representatives for the cosets of $G^{(i)}$ in $G^{(i-1)}(2 \leq i \leq k)$, then each element of $G$ can be uniquely expressed as a product $u_{k-1} u_{k-2} \ldots u_{1}$, where $u_{i} \in U^{(i)}(1 \leq i<k)$ [3]. Permutations can be generated incrementally using elements from the coset representatives, and the set of images of a state $s$ under $G$ computed using a sequence of partial images (see Algorithm 2). To ensure efficient application of permutations, the coset representatives are stored as a list of transpositions, applied in succession. GAP provides functionality to efficiently compute a stabiliser chain and associated coset representatives for an arbitrary permutation group. This approach still involves enumeration of $G$, so is infeasible for large groups, but the experimental results of Section 5.3 show an improvement over basic enumeration. Additionally, it is only necessary to store coset representatives, rather than all elements of $G$.

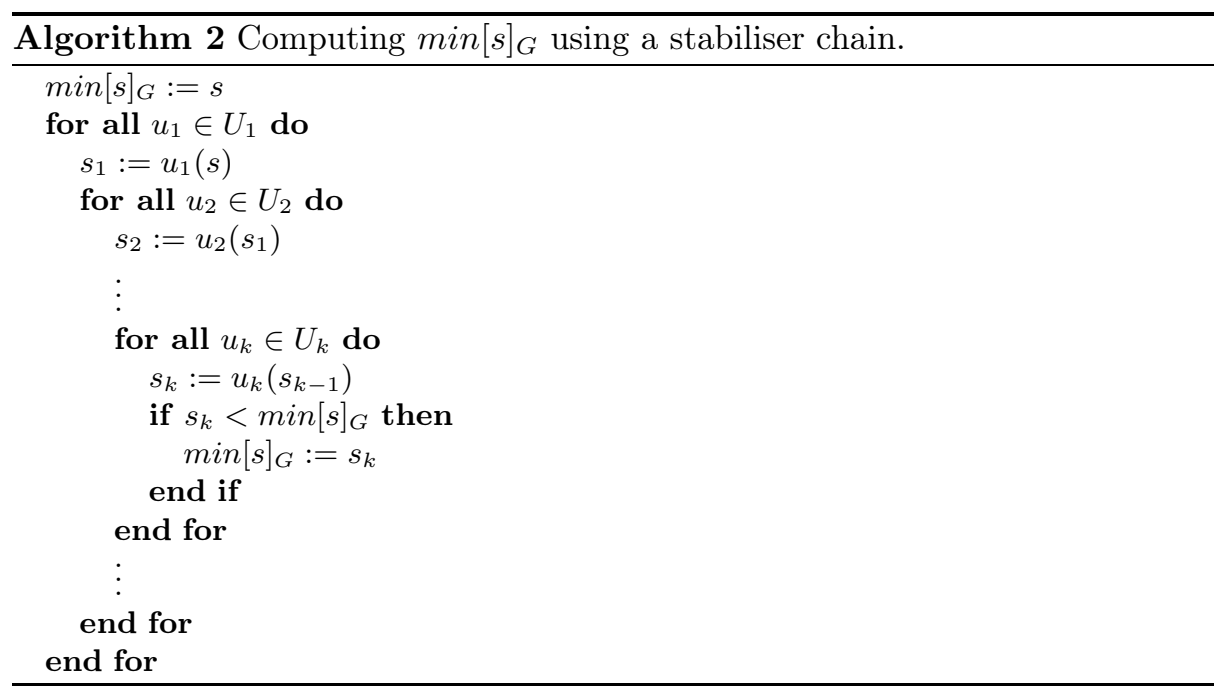

\subsection{Minimising Sets for $G$ if $G \cong S_{m}(m \leq n)$}

For systems where there is full symmetry between components, the smallest state in the orbit of $s=\left(l_{1}, l_{2}, \ldots, l_{n}\right)$ can be computed by sorting the tuple $\left(l_{1}, l_{2}, \ldots, l_{n}\right)$ under the lexicographical ordering so that the smallest $l_{i}$ appears in the first position, the second smallest in the second position etc. $[2,5]$. For example, for a system with four components, sorting equivalent states $(3,2,1,3)$ and $(3,3,2,1)$ yields the state $(1,2,3,3)$, which is clearly the smallest state in the orbit. Since sorting can be performed in polynomial time, this provides an efficient solution for the COP for this group. 
Recall the group $G_{3 T}$ of automorphisms of the communication graph of Figure 1. Consider the subgroup

$$
H=\langle(12 \text { 13)(1 4)(2 5)(3 6),(13 14)(4 7)(5 8)(6 9)〉. }
$$

This group permutes server components 12, 13 and 14, with their associated blocks of client components. It is clear that $H$ is isomorphic to $S_{3}$, the symmetric group on 3 objects. However, we cannot compute $\min [s]_{H}$ by sorting $s$ in the obvious way, since this is equivalent to applying an element $\alpha \in S_{16}$ to $s$, which may not belong to $H$.

In some cases we can deal with groups of this form using a minimising set for $G$. Using terminology from [11], $G$ is said to be nice if there is a small set $X \subseteq G$ such that, for any $s \in S, s=\min [s] \Leftrightarrow s \leq \alpha(s) \forall \alpha \in X$. If $G$ is nice with respect to a subset $X$, then we call $X$ a minimising set for $G$. In this case, $\min [s]_{G}$ can be computed using Algorithm 3. If a small minimising set $X$ can be found for a large group $G$, then computing the representative of a state involves iterating over the small set $X$, minimising the state until a fix-point is reached. At this point, no element of the minimising set maps the state to a smaller image, thus the minimal element has been found.

We show that, for a large class of groups which are isomorphic to $S_{m}$ for some $m \leq n$, a minimising set with size polynomial in $m$ can be efficiently found. This minimising set is derived from the swap permutations used in a selection sort algorithm.

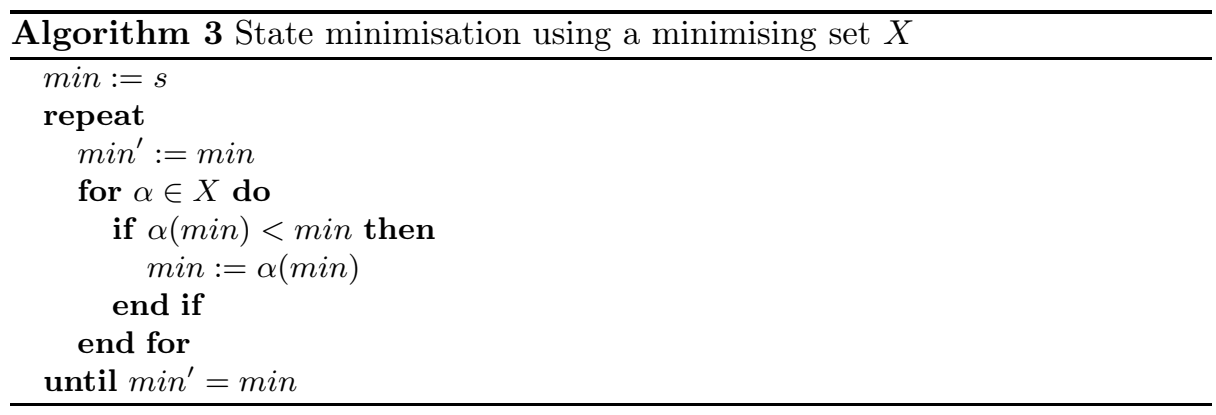

Theorem 2. Suppose that, for each $i \in I$ such that $\operatorname{orb}_{G}(i)$ is non-trivial, $s^{\text {stab }}{ }_{G}(i)$ fixes exactly one element from each non-trivial orbit of $G$ acting on $I$, and that $G \cong S_{m}$, where $m=\left|\operatorname{orb}_{G}(i)\right|>1$ for some $i \in I$. Then there is an isomorphism $\theta: S_{m} \rightarrow G$ such that $\left\{(i j)^{\theta}: 1 \leq i<j \leq m\right\}$ is a minimising set for $G$.

Proof. Since for each $i \in I$ such that $\left|\operatorname{orb}_{G}(i)\right|>1$ the set of elements fixed by $\operatorname{stab}_{G}(i)$ contains exactly one element from each orbit, there is a set of columns $C_{1}, C_{2}, \ldots, C_{m}$ such that each column contains one element from each orbit of $\mathrm{G}$, and $\mathrm{G}$ permutes the columns. There is an isomorphism $\theta$ from $G^{\prime}$ (the action 
of $G$ on the columns) to $G$ acting on $I$. Since $G \cong S_{m}, G^{\prime}$ contains all column transpositions $(i j)$ where $i<j$, so $(i j)^{\theta} \in G$. The element $(i j)^{\theta}$ maps all elements of column $i$ to elements of column $j$.

Now consider states $s$ and $s^{\prime}$, where $s^{\prime}=\alpha(s)$ for some $\alpha \in G$. Let $i$ be the smallest index for which $s(i) \neq s^{\prime}(i)$. Let $j$ be the index such that $j=\alpha^{-1}(i)$. All of the elements in the column containing $j$ (column $j^{\prime}$ say) are mapped via $\alpha$ to the column containing $i$ (column $i^{\prime}$ say). Then $s^{\prime}<s$ iff $\left(i^{\prime} j^{\prime}\right)^{\theta} s<s$. Hence $s$ is minimal in its orbit iff $(i j)^{\theta}(s) \geq s$ for all $i<j$. So the set $\left\{(i j)^{\theta}: 1 \leq i<\right.$ $j \leq m\}$ is a minimising set for $G$.

Note that the minimising set is much smaller than $G$, and the conditions of Theorem 2 can be easily checked using GAP. It may seem that these conditions are unnecessary, and that, given any isomorphism $\theta: S_{m} \rightarrow G$, the set $\left\{(i j)^{\theta}\right.$ : $1 \leq i<j \leq m\}$ is a minimising set for $G$. However, the group

$$
G=\langle(12)(56)(910)(1314),(1248)(36129)(510)(7141311)\rangle \leq S_{14}
$$

is isomorphic to $S_{4}$. An isomorphism $\theta: S_{4} \rightarrow G$ is defined on generators by

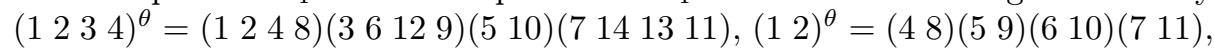
and can be shown to be the only isomorphism between these groups. The state $s=(8,14,4,8,4,7,9,14,6,10,3,1,11,4) \in\{1,2, \ldots, 14\}^{14}$ cannot be minimised using the set $\left\{(i j)^{\theta}: 1 \leq i<j \leq 4\right\}$. The group $G$ is a subgroup of the symmetry group of a hypercube (see Section 5.3). However, Theorem 2 allows us to find minimising sets for commonly occurring fully symmetric groups.

\subsection{Local Search for Unclassifiable Groups}

If $G$ is large group then computing $\min [s]_{G}$ by enumeration of the elements of $G$ may be infeasible, even with the group-theoretic optimisations discussed in Section 3.1. If no minimising set is available for $G$, and $G$ cannot be classified as a composite symmetry group (see Section 4 ) then we must exploit $G$ via an approximate symmetry reduction strategy.

We propose an approximate strategy based on hillclimbing local search, which has proved successful for a variety of search problems in artificial intelligence [18, Chapter 4]. The function min works by performing a local search of $[s]_{G}$ starting at $s$, using the generators of $G$ as operations from which to compute a successor state. The search starts by setting $t=s$, and proceeds iteratively. On each iteration, $\alpha(t)$ is computed for each generator $\alpha$ of $G$. If $t \leq \alpha(t)$ for all $\alpha$ then a local minimum has been reached, and $t$ is returned as a representative for $[s]_{G}$. Otherwise, $t$ is set to the smallest image $\alpha(t)$, and the search continues. In Section 5.3 we show that this local search algorithm is effective when exploring the state spaces of various configurations of message routing in a hypercube network.

There are various local search techniques which could be employed to attempt to improve the accuracy of this strategy. Random-restart hill-climbing [18] works by picking several random elements of $[s]_{G}$ in addition to $s$, and performing local 
search from each of them, returning the smallest result. This could be achieved by applying distinct, random elements of $G$ to $s$ (GAP provides functionality for generating random group elements). Another potential improvement would be to use simulated annealing [17] to escape local minima.

\section{Exploiting Composite Symmetry Groups}

Certain kinds of symmetry groups can be decomposed as a product of subgroups. In this case solving the COP separately for each subgroup provides a solution to the COP for the whole group. In particular, if a symmetry group permutes disjoint sets of components independently then the group can be described as the disjoint product of the groups acting on these disjoint sets. On the other hand, if the symmetry group partitions the components into subsets such that there is analogous symmetry within each subset, and symmetry between the subsets, then the group can be described as the wreath product of the group which acts on one of the subsets, and the group which permutes the subsets. It has been shown that, if $G$ is known to be a disjoint or wreath product of subgroups, then the COP can be solved for $G$ by restricting attention to these subgroups [5]. We now present solutions to the problem of detecting, before search, whether or not $G$ can be decomposed.

\subsection{Disjoint Products}

Definition 1. Let $H \leq S_{n}$. Suppose that $H_{1}, H_{2}, \ldots, H_{k}$ are subgroups of $H$ $(1 \leq i \leq k, k>1)$. If $H=H_{1} H_{2} \ldots H_{k}=\left\{\alpha_{1} \alpha_{2} \ldots \alpha_{k}: \alpha \in H_{i}(1 \leq i \leq k)\right\}$ then $H$ is called the product of the $H_{i}$. If $\operatorname{moved}\left(H_{i}\right) \cap \operatorname{moved}\left(H_{j}\right)=\emptyset$ for all $1 \leq i \neq j \leq k$ then $H$ is written $H_{1} \bullet H_{2} \bullet \cdots \bullet H_{k}$, and called the disjoint product of the $H_{i}$. The disjoint product is said to be non-trivial if $H \neq H_{i} \neq\{i d\}$ for all $1 \leq i \leq k$.

Disjoint products occur frequently in model checking problems. For example, the symmetry group associated with a model of the readers writers problem [10] may be a disjoint product of two groups, which independently permute reader and writer components respectively. In our three-tiered architecture example (see Section 2.4), the group $G_{3 T}$ can be shown to decompose as a disjoint product $G_{3 T}=H_{1} \bullet H_{2}$ where:

$$
\begin{aligned}
H_{1}= & \langle(12),(23),(45),(56),(78),(89), \\
& (1213)(14)(25)(36),(1314)(47)(58)(69)\rangle \\
H_{2}= & \langle(10,11)\rangle .
\end{aligned}
$$

If $G$ is a disjoint product of subgroups $H_{1}, H_{2}, \ldots, H_{k}$ then $\min [s]_{G}=\min [\ldots$ $\left.\min \left[\min [s]_{H_{1}}\right]_{H_{2}} \ldots\right]_{H_{k}}[5]$, so the COP for $G$ can be solved by considering each subgroup $H_{i}$ in turn. This result is only useful when designing a fully automatic symmetry reduction package if it is possible to efficiently determine, 
before search, whether or not $G$ decomposes as a disjoint product of subgroups. We present two solutions to this problem.

\section{Efficient, sound, incomplete approach}

Let $G=\langle X\rangle$ for some $X \subseteq G$ with $i d \notin X$. Define a binary relation $B \subseteq X^{2}$ as follows: for all $\alpha, \beta \in X,(\alpha, \beta) \in B \Leftrightarrow \operatorname{moved}(\alpha) \cap \operatorname{moved}(\beta) \neq \emptyset$. Clearly $B$ is symmetric, and since for any $\alpha \in G$ with $\alpha \neq i d, \operatorname{moved}(\alpha) \neq \emptyset$, reflexive. It follows that the transitive closure of $B$, denoted $B^{*}$, is an equivalence relation on $X$. We now show that if $B^{*}$ has multiple equivalence classes then each class generates a subgroup of $G$ which is a non-trivial factor for a disjoint product decomposition of $G$.

Lemma 1. Suppose that $\alpha, \beta \in X$, and that $(\alpha, \beta) \notin B^{*}$. Then moved $(\alpha) \cap$ $\operatorname{moved}(\beta)=\emptyset$ and $\alpha$ and $\beta$ commute.

Theorem 3. Suppose $C_{1}, C_{2}, \ldots, C_{k}$ are the equivalence classes of $X$ under $B^{*}$ where $k \geq 2$. For $1 \leq i \leq k$ let $H_{i}=\left\langle C_{i}\right\rangle$. Then $G=H_{1} \bullet H_{2} \bullet \cdots \bullet H_{k}$, and $H_{i} \neq\{i d\}(1 \leq i \leq k)$.

Proof. Clearly $H_{1} H_{2} \ldots H_{k} \subseteq G$. If $\alpha \in G$ then $\alpha=\alpha_{1} \alpha_{2} \ldots \alpha_{d}$ for some $\alpha_{1}, \alpha_{2}, \ldots, \alpha_{d} \in X, d>0$. By Lemma 1 we can arrange the $\alpha_{l}$ so that elements of $C_{i}$ appear before those of $C_{j}$ whenever $i<j$. It follows that $G=$ $H_{1} H_{2} \ldots H_{k}$. By Lemma $1, \operatorname{moved}\left(H_{i}\right) \cap \operatorname{moved}\left(H_{j}\right)=\emptyset$ for $1 \leq i \neq j \leq k$ and so $G=H_{1} \bullet H_{2} \bullet \cdots \bullet H_{k}$, where (since $i d \notin X$ ) the $H_{i}$ are non-trivial.

The approach is incomplete as it does not guarantee the finest decomposition of $G$ as a disjoint product. However, in practice we have not found a case in which the finest decomposition is not detected when generators have been computed by a graph automorphism program. The approach is very efficient as it works purely with the generators of $G$, of which there are typically few.

\section{Sound and complete approach}

It is straightforward to show that if $G=H \bullet K$, then $H$ and $K$ are normal subgroups of $G$. Thus a complete method for determining whether or not $G$ is a non-trivial disjoint product of subgroups $H$ and $K$ involves the computation of all normal subgroups of $G$ and searching for a pair such that $G=H \bullet K$. This method could be applied recursively to the factors of the disjoint product to compute the finest disjoint product decomposition of $G$. Although for certain groups (e.g. abelian groups), all subgroups are normal, in many cases the number of normal subgroups of a group is significantly smaller than the number of arbitrary subgroups.

\subsection{Wreath Products}

Definition 2. For $r>1$ let $B_{1}, B_{2}, \ldots, B_{r}$ be disjoint subsets of $I$, where $B_{i}=$ $\left\{b_{i, 1}, b_{i, 2}, \ldots, b_{i, m}\right\}$ for some $m>1$. Let $H \leq S_{n}$ with moved $(H) \subseteq B_{1}$. For any $\alpha \in H$ and $1 \leq i \leq r$, define $\alpha^{(i)}$ by: $\alpha^{(i)}(x)=x$ if $x \notin B_{i} ; \alpha^{(i)}\left(b_{i, j}\right)=b_{i, l}$ 
where $\alpha\left(b_{1, j}\right)=b_{1, l}$. For $\beta^{\prime} \in S_{r}$, define $\beta \in S_{n}$ by: $\beta(x)=x$ if $x \notin \bigcup_{1 \leq i \leq r} B_{i}$; $\beta\left(b_{i, j}\right)=b_{\beta^{\prime}(i), j}$. Let $K=\left\langle\beta_{1}, \beta_{2}, \ldots, \beta_{d}\right\rangle$ where $\beta_{i}^{\prime} \in S_{k}(1 \leq i \leq d, d>0)$. If every element of $G$ can be expressed in the form $\beta \alpha_{r}^{(r)} \ldots \alpha_{2}^{(2)} \alpha_{1}^{(1)}$, where $\beta \in K$ and $\alpha_{1}, \alpha_{2}, \ldots, \alpha_{r} \in H$, then $G$ is the wreath product of $H$ and $K$, denoted $H$ 乙 K.

Intuitively, an element of $G$ applies a permutation to each set $B_{i}$, then applies a permutation which permutes the sets. This definition of wreath products is specific to those that occur in model checking problems, typically when a system has a tree structure. For a more general definition, see [4]. In Section 4.1, we showed that the group $G_{3 T}$ decomposes as a disjoint product. Consider the factor $H_{1}$ of this product. This group itself decomposes as a wreath product $H_{1}=H \imath K$ where:

$$
\begin{aligned}
H & =\langle(12),(23)\rangle \\
K & =\langle(1213)(14)(25)(36),(1314)(47)(5 \text { 8) }(69)(10,11)\rangle .
\end{aligned}
$$

Here, the sets are $B_{1}=\{1,2,3,12\}, B_{2}=\{4,5,6,13\}$ and $B_{3}=\{7,8,9,14\}$. If $G=H \imath K$ then, for $1 \leq i \leq k$ define $H_{i}$ by $\left\{\alpha^{(i)}: \alpha \in K\right.$. Then $\min [s]_{G}=$ $\min \left[\min \left[\ldots \min \left[\min [s]_{H_{1}}\right]_{H_{2}} \ldots\right]_{H_{r}}\right]_{K}[5]$.

We sketch an approach for detecting whether an arbitrary group is a wreath product of subgroups. If $G$ acts transitively on $I$, a subset $B$ of $I$ is a block if, for any $\alpha \in G, \alpha(B)=B$ or $B \cap \alpha(B)=\emptyset$. The set $\mathcal{B}=\{\alpha(B): \alpha \in G\}$ is a block system for $G$. Given block systems $\mathcal{B}, \mathcal{C}$ for $G, \mathcal{C}$ is strictly coarser than $\mathcal{B}$ if $\forall B \in \mathcal{B} \exists C \in \mathcal{C}$ such that $B \subset C$, and $\mathcal{B}$ is maximal for $G$ if each $B \in \mathcal{B}$ is a proper subset of $I$, and the only block system strictly coarser than $\mathcal{B}$ is the trivial system $\{I\}$.

If $\left\{B_{1}, B_{2}, \ldots, B_{r}\right\}$ is a block system for a transitive group $G$, where $B_{i}=$ $\left\{b_{i, 1}, b_{i, 2}, \ldots, b_{i, m}\right\}$ then $G \leq H<K$, where $H=\bigcap_{i \notin B_{1}} \operatorname{stab}_{G}(i)$, and $K=$ $\bigcap_{1 \leq i \leq m} \operatorname{stab}_{G}\left(\left\{b_{1, i}, b_{2, i}, \ldots, b_{r, i}\right\}\right)$ [4]. To check whether or not $G=H\langle K$ it is sufficient to compare orders, and it can be shown that $|H \curlywedge K|=|H|^{r}|K|$. However, in general $G$ does not act transitively on $I$. We solve the general problem of determining whether or not $G$ is a wreath product of subgroups by considering the action of $G$ separately on each non-trivial orbit of $I$.

Lemma 2. If $G=H \nmid K$ then each non-trivial orbit $O$ of $I$ under $G$ has a single maximal block system: $\left.\left\{O \cap \operatorname{moved}\left(H_{i}\right): 1 \leq i \leq r\right)\right\}$.

If $G$ can be shown to have exactly one maximal block system per orbit, then candidate groups $H$ and $K$ can be constructed. Suppose the non-trivial orbits are $O_{1}, O_{2}, \ldots, O_{d}$. For $1 \leq i \leq d$, the group $K_{i}$ is computed as follows: $K_{i}=\bigcap_{1 \leq i \leq m} \operatorname{stab}_{G}\left(\left\{b_{1, i}, b_{2, i}, \ldots, b_{r, i}\right\}\right)$, where the elements $b_{i, j}$ are taken from the maximal block system for $G$ on $O_{i}$. The candidate group $K$ is the intersection of the $K_{i}$. Candidate group $H$ is initially set to $G$. For each orbit $O_{i}$ a maximal block $B$ is chosen such that $B \subseteq \operatorname{moved}(H)$. Then $H$ is recomputed as $\bigcap_{i \in O_{i} \backslash B} \operatorname{stab}_{H}(i)$. We now have groups $H$ and $K$ with $G \leq H \curlywedge K$, and we can check whether $G=H \imath K$ by comparing orders, as before. 


\subsection{Choosing a Strategy for $G$}

The strategies which we have presented for minimising a state with respect to basic and composite groups can be combined to yield a symmetry reduction strategy for the arbitrary group $G$ by classifying the group using a top-down recursive algorithm.

The algorithm starts by searching for a minimising set for $G$ of the form prescribed in Theorem 2. Such a minimising set means that $\min [s]_{G}$ can be computed using Algorithm 3. If no such minimising set can be found, a decomposition of $G$ as a disjoint/wreath product is sought. In this case the algorithm is applied recursively to obtain a minimisation strategy for each factor of the product so that $\min [s]_{G}$ can be computed using these strategies as described in Sections 4.1 and 4.2 respectively. If $G$ remains unclassified and $|G| \leq n^{2}$ say, where $n$ is the number of components, enumeration is used, otherwise local search is selected.

\section{Symmetry Reductions in Practice}

\subsection{Extending the Model of Computation}

When components do not hold references to other components, the simple model of computation and the action of a permutation on a state (described in Sections 2.1 and 2.3 respectively) are sufficient to reason about concurrent systems, since it is always possible to represent the local state of a component using an integer. However, if components can hold references to one another then any permutation that moves component $i$ will also affect the local state of any components which refer to component $i$.

Sophisticated specification languages, such as Promela, include special datatypes to represent process and channel identifiers. An extended model of computation for Promela is presented in [8]. Both the results presented in [5] on solving the COP for groups which decompose as disjoint/wreath products, and our results on minimising sets for fully symmetric groups (see Section 3.2) do not hold in general for this extended model of computation.

Thus for Promela specifications where local variables refer to process and channel identifiers, the efficient symmetry reduction strategies presented above are not always exact - in some cases they may yield an approximate implementation of the function min, as discussed in Section 2.3. This does not compromise the safety of symmetry reduced model checking, and in any case, for a large model, there will be many states for which the strategies will give exact representatives in an extended model of computation as the experimental results in Section 5.3 show.

For the simple case of full symmetry between identical components, the SymmSpin package deals with local variables which are references to component identifiers by dividing the local state of each component into two portions, one which does not refer to other components (the insensitive portion say), and another which consists entirely of such references (the sensitive portion). A state 
is minimised by first sorting it with respect to the insensitive portion. Then, for each subset of components with identical insensitive portions, every permutation of the subset is considered, and the permutation which leads to the smallest image is applied. This is known as the segmented strategy. Our approach using minimising sets is similar to the sorted strategy which SymmSpin also provides. Here a state is minimised only with respect to the insensitive portions of the local states. This strategy is much faster than the segmented strategy, but is approximate. It may be possible to extend our approach to be exact by generalising the segmented strategy.

\subsection{A Symmetry Reduction Package for SPIN}

We have implemented the strategies discussed in Sections 3 and 4 as TopSPIN, a fully automatic symmetry reduction package for SpIN [9]. In order to check properties of a Promela specification, SPIN first converts the specification into a C source file, pan.c, which is then compiled into an executable verifier. The state space thus generated is then searched. If the property being checked is proved to be false, a counterexample is given. TopSPIN follows the approach used by the SymmSpin symmetry reduction package, where pan.c is generated as usual by SPIN, and then converted to a new file, sympan.c, which includes algorithms for symmetry reduction. With TopSPIN, because we allow for arbitrary system topologies, symmetry must be detected before sympan.c can be generated. This is illustrated in Figure 2.

First, the static channel diagram (SCD) of the Promela specification is extracted by the SymmExtractor tool, which is described in detail in [8]. The SCD is a graphical representation of potential communication between components of the specification. The group of symmetries of the SCD, Aut $(S C D)$, is computed using the saucy tool [7], which we have extended to handle directed graphs. The generators of $A u t(S C D)$ are checked against the Promela specification for validity (an assurance that they induce symmetries of the underlying state space). TopSPIN uses GAP to compute, from the set of valid generators, the largest group $G \leq A u t(S C D)$ which can be safely used for symmetry-reduced model checking. GAP is then used to classify the structure of $G$ in order to choose an efficient symmetry reduction strategy. The chosen strategy is merged with pan.c to form sympan.c, which can be compiled and executed as usual. In order to compare strategies it is possible to select the strategy used (rather than let TopSPIN choose the most efficient). For experimental purposes, TopSpin also allows generators of an arbitrary group of component symmetries to be specified manually, as long as the group elements do not permute components with different types.

\subsection{Experimental Results}

In Table 1 we present experimental results applying TopSpin to four families of Promela specifications. For each specification, we give the number of model states without symmetry reduction (orig), with full symmetry reduction (red), and using the strategy chosen by TopSPIN (best). If the latter two are equal, 


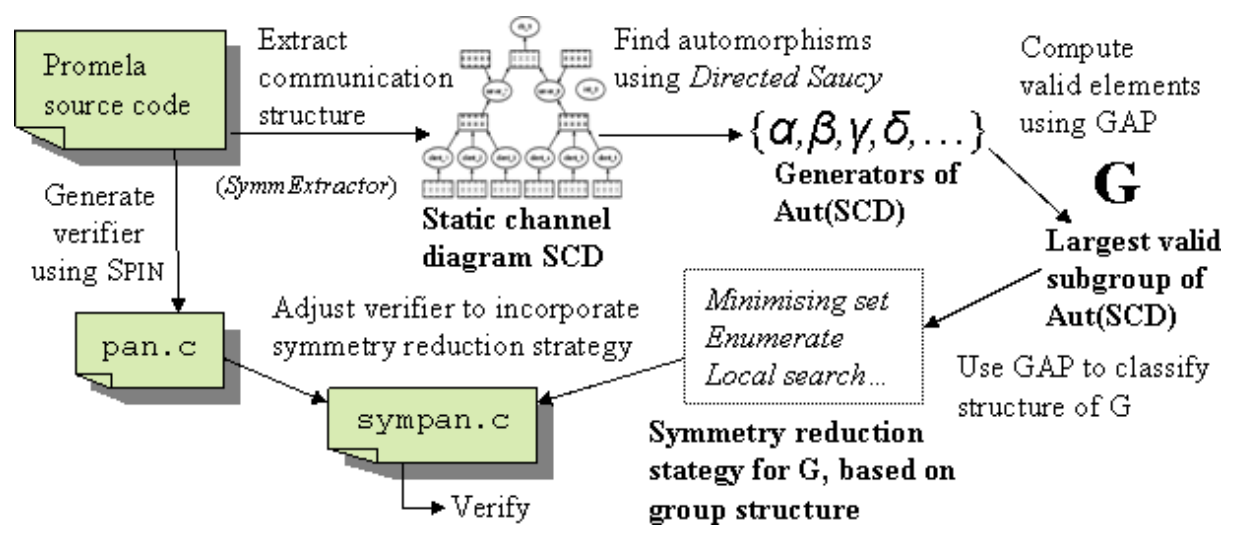

Fig. 2. The symmetry reduction process.

'=' appears for the TopSPIN strategy. The use of state compression, provided by SPIN, is indicated by the number of states in italics. For each strategy (basic for enumeration without the optimisations described in Section 3.1, enum for optimised enumeration, and best for the strategy chosen by TopSPIN), and when symmetry reduction is not applied (orig), we give the time taken for verification (in seconds). Verification attempts which exceed available resources, or do not terminate within 5 hours, are indicated by '-'. All experiments are performed on a PC with a $2.4 \mathrm{GHz}$ Intel Xeon processor, $3 \mathrm{~Gb}$ of available main memory, running SPIN version 4.2.3.

The first family of specifications model flow of control for a system similar to the three-tiered architecture example of Section 2.4, but with a layer of $p$ servers with $q$ clients connected to each server (a D-S-C system). Here models exhibit wreath product symmetry: there is full symmetry between the $q$ clients in each block, and the blocks of clients, with their associated servers, are interchangeable. A configuration with $p$ servers and $q$ clients per server is denoted $p / q$. The second family of specifications model a resource allocator process which controls access to a resource by a competing set of prioritised clients (an R-C system). Models of these specifications exhibit disjoint product symmetry: there is full symmetry between each set of clients with the same priority level. A configuration with $p_{i}$ clients of priority level $i$ is denoted $p_{1}, \ldots, p_{k}$, where $k$ is the number of priority levels. The next family consists of specifications which model an email system where client processes exchange messages via a mailer process. The symmetries of models of these specifications permute the client processes, simultaneously permuting their input channels, and can be handled using a minimising set. A configuration with $p$ clients is simply denoted $p$. Finally, we consider specifications modelling message routing in an $n$ dimensional hypercube network (an $\mathrm{HC}$ system). The symmetry group here is isomorphic to the group of geometrical symmetries of a $n$ dimensional hypercube, which cannot be decomposed as a 
product of subgroups, and thus must be handled using either the enumeration or local search strategies. An $n$-dimensional hypercube specification is denoted $n$ d. For all specifications, we verify deadlock freedom, and check the satisfaction of basic safety properties expressed using assertions.

\begin{tabular}{|c|c|c|c|c|c|c|c|c|c|}
\hline system & config. & $\begin{array}{c}\text { states } \\
\text { orig }\end{array}$ & $\begin{array}{c}\text { time } \\
\text { orig }\end{array}$ & $|G|$ & $\begin{array}{c}\text { states } \\
\text { red }\end{array}$ & $\begin{array}{c}\text { time } \\
\text { basic }\end{array}$ & $\begin{array}{c}\text { time } \\
\text { enum }\end{array}$ & $\begin{array}{c}\text { states } \\
\text { best }\end{array}$ & $\begin{array}{c}\text { time } \\
\text { best }\end{array}$ \\
\hline \hline D-S-C & $2 / 3$ & 103105 & 5 & 72 & 2656 & 7 & 4 & $=$ & 2 \\
\hline D-S-C & $2 / 4$ & $1.1 \times 10^{6}$ & 37 & 1152 & 5012 & 276 & 108 & $=$ & 2 \\
\hline D-S-C & $3 / 3$ & $2.54 \times 10^{7}$ & 4156 & 1296 & 50396 & 4228 & 1689 & $=$ & 19 \\
\hline D-S-C & $3 / 4$ & - & - & 82944 & - & - & - & 130348 & 104 \\
\hline \hline R-C & 3,3 & 16768 & 0.2 & 36 & 1501 & 0.9 & 0.3 & $=$ & 0.1 \\
\hline R-C & 4,4 & 199018 & 2 & 576 & 3826 & 57 & 19 & $=$ & 0.4 \\
\hline R-C & 5,5 & $2.2 \times 10^{6}$ & 42 & 14400 & 8212 & 4358 & 1234 & $=$ & 2 \\
\hline R-C & $4,4,4$ & $2.39 \times 10^{7}$ & 1587 & 13824 & 84377 & - & 12029 & $=$ & 17 \\
\hline R-C & $5,5,5$ & - & - & 1728000 & - & - & - & 254091 & 115 \\
\hline \hline email & 3 & 23256 & 0.1 & 6 & 3902 & 0.9 & 0.8 & 3908 & 0.2 \\
\hline email & 4 & 852641 & 9 & 24 & 36255 & 13 & 6 & 38560 & 2 \\
\hline email & 5 & $3.04 \times 10^{7}$ & 3576 & 120 & 265315 & 679 & 253 & 315323 & 40 \\
\hline email & 6 & - & - & 720 & $1.7 \times 10^{6}$ & - & 13523 & $2.3 \times 10^{6}$ & 576 \\
\hline email & 7 & - & - & 5040 & - & - & - & $1.53 \times 10^{7}$ & 6573 \\
\hline \hline HC & $3 \mathrm{~d}$ & 13181 & 0.3 & 48 & 308 & 0.6 & 0.3 & 468 & 0.2 \\
\hline HC & $4 \mathrm{~d}$ & 380537 & 18 & 384 & 1240 & 58 & 34 & 6986 & 13 \\
\hline HC & $5 \mathrm{~d}$ & $9.6 \times 10^{6}$ & 2965 & 3840 & 3907 & 7442 & 5241 & 90442 & 946 \\
\hline
\end{tabular}

Table 1. Experimental results for various configurations of the three-tiered (D-S-C), resource allocator (R-C), email (email) and hypercube (HC) specifications

In all cases, the basic enumeration strategy is significantly slower than the optimised enumeration strategy, which is in turn slower than the strategies chosen by TopSpin. For the three-tiered and resource allocator configurations the symmetry reduction strategies chosen by TopSpin, which decompose the symmetry group as a wreath/disjoint product of groups which are then handled by minimising sets, provide exact symmetry reduction, despite the potential problems discussed in Section 5.1. This is not the case for email configurations, for which TopSpin uses minimising sets. Nevertheless, a large factor of reduction is gained by exploiting symmetry in this way, and verification is fast. For hypercube configurations, TopSpIN chooses the local search strategy, which requires storage of more states than the enumeration strategy, but still results in a greatly reduced state space. 


\section{Related Work}

The simple model of computation which we have used throughout the paper is common to numerous works on symmetry reductions for model checking, for example [5,10-12], and is adequate for reasoning about input languages where components do not individually hold references to other components, e.g. the input languages of SMC [19], SYMM [5] and UTOOL [12], or where components are specified using synchronisation skeletons [10]. The problem of extending symmetry reduction techniques to a model of computation where such references are allowed is tackled, for the simple case of full symmetry between identical components, by the segmented strategy of the SymmSpin package.

Methods for exploiting the disjoint/wreath product structure of symmetry groups were proposed in [5], but this work did not investigate the problem of classifying the structure of an arbitrary group, as we have done. Stabiliser chains (see Section 3.1) are used extensively in computational group theory [3,13], and have been utilised in symmetry breaking approaches for constraint programming [14]. This paper is, to our knowledge, the first to apply these techniques to model checking. The construction of minimising sets for fully symmetric groups which we presented in Section 3.2 builds on the concept of a nice group [11], and generalises the idea of computing orbit representatives by sorting $[2,5,11]$.

\section{Conclusions and Future Work}

In this paper, we have proposed exact and approximate strategies for tackling the NP-hard problem of computing orbit representatives in order to exploit symmetry when model checking concurrent systems, and generalised existing results in this area. We have applied techniques from computational group theory to speed up representative computation, and to classify the structure of a symmetry group as a disjoint/wreath product of subgroups before search. We have described TopSpIN, a fully automatic symmetry reduction package for SPIN, and presented encouraging experimental results for a variety of Promela specifications.

We are currently investigating further the use of local search techniques as an approximate symmetry reduction strategy. We are also developing an approach to generalise the segmented strategy used by the SymmSpin package to overcome potential inefficiencies associated with extending our simple model of computation to the Promela language. TopSPIN is currently limited to verifying the absence of deadlock and the satisfaction of safety properties of Promela specifications. Future work includes extending TopSPIN to allow symmetry-reduced verification of temporal properties with weak fairness, as described in [1].

\section{References}

1. D. Bosnacki. A light-weight algorithm for model checking with symmetry reduction and weak fairness. In SPIN'03, LNCS 2648, pages 89-103. Springer, 2003. 
2. D. Bosnacki, D. Dams, and L. Holenderski. Symmetric spin. International Journal on Software Tools for Technology Transfer, 4(1):65-80, 2002.

3. G. Butler. Fundamental Algorithms for Permutation Groups, volume 559 of LNCS. Springer-Verlag, 1991.

4. P.J. Cameron. Permutation Groups. Cambridge University Press, 1999.

5. E.M. Clarke, E.A. Emerson, S. Jha, and A.P. Sistla. Symmetry reductions in model checking. In $C A V^{\prime} 98$, LNCS 1427, pages 147-158. Springer, 1998.

6. E.M. Clarke, O. Grumberg, and D. Peled. Model Checking. The MIT Press, 1999.

7. P.T. Darga, M.H. Liffiton, K.A. Sakallah, and I.L. Markov. Exploiting structure in symmetry detection for CNF. In $D A C^{\prime} 04$, pages 530-534. ACM Press, 2004.

8. A.F. Donaldson and A. Miller. Automatic symmetry detection for model checking using computational group theory. In FM'05, LNCS 3582, pages 418-496. Springer, 2005.

9. A.F. Donaldson and A. Miller. A computational group theoretic symmetry reduction package for the SPIN model checker. In $A M A S T^{\prime} 06$, LNCS 4019, pages 374-380. Springer, 2006.

10. E.A. Emerson and R.J. Trefler. From asymmetry to full symmetry: new techniques for symmetry reduction in model checking. In CHARME'99, LNCS 1703, pages 142 156. Springer, 1999.

11. E.A. Emerson and T. Wahl. Dynamic symmetry reduction. In TACAS' 05 , LNCS 3440, pages 382-396. Springer, 2005.

12. E.A. Emerson and T. Wahl. Efficient reduction techniques for systems with many components. Electronic Notes in Theoretical Computer Science, 130:379-399, 2005.

13. The Gap Group. GAP-Groups Algorithms and Programming, Version 4.2. Aachen, St. Andrews, 1999. http://www-gap.dcs.st-and.ac.uk/ gap.

14. I.P. Gent, W. Harvey, and T. Kelsey. Groups and constraints: symmetry breaking during search. In CP'02, LNCS 2470, pages 415-430. Springer, 2002.

15. G. J. Holzmann. The SPIN model checker: primer and reference manual. Addison Wesley, 2003.

16. C.N. Ip and D.L. Dill. Better verification through symmetry. Formal Methods in System Design, 9(1/2): 41-75, 1996.

17. K.S. Kirkpatrick, C.D. Gelatt, and M.P. Vecchi. Optimization by simulated annealing. Science, 220: 671-680, 1983.

18. S. Russel and P. Norvig. Artificial Intelligence, a Modern Approach. Prentice Hall, 1995.

19. A.P. Sistla, V. Gyuris, and E.A. Emerson. SMC: a symmetry-based model checker for verification of safety and liveness properties. ACM Transactions on Software Engineering and Methodology, 9(2):113-166, 2000. 